\title{
Besicovitch-Federer projection theorem for continuously differentiable mappings having constant rank of the Jacobian matrix
}

\author{
Jacek Gałęski ${ }^{1}$
}

Received: 10 April 2017 / Accepted: 30 August 2017 / Published online: 10 November 2017

(C) The Author(s) 2017. This article is an open access publication

\begin{abstract}
The purpose of this article is to prove a generalisation of the Besicovitch-Federer projection theorem about a characterisation of rectifiable and unrectifiable sets in terms of their projections. For an $m$-unrectifiable set $\Sigma \subset \mathbb{R}^{n}$ having finite Hausdorff measure and $\varepsilon>0$, we prove that for a mapping $f \in \mathscr{C}^{1}\left(U, \mathbb{R}^{n}\right)$ having constant, equal to $m$, rank of the Jacobian matrix there exists a mapping $f_{\varepsilon}$ whose rank of the Jacobian matrix is also constant, equal to $m$, such that $\left\|f_{\varepsilon}-f\right\|_{\mathscr{C} 1}<\varepsilon$ and $\mathscr{H}^{m}\left(f_{\varepsilon}(\Sigma)\right)=0$. We derive it as a consequence of the Besicovitch-Federer theorem stating that the $\mathscr{H}^{m}$ measure of a generic projection of an $m$-unrectifiable set $\Sigma$ onto an $m$-dimensional plane is equal to zero.
\end{abstract}

Keywords Purely unrectifiable set · Hausdorff measure $\cdot$ Rank of the Jacobian matrix

Mathematics Subject Classification Primary 28A75; Secondary 57N20

\section{Contents}

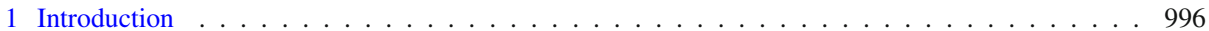

1.1 Notation and conventions . . . . . . . . . . . . . . . . . . . . . . . . . . . . 998

2 Local variant . . . . . . . . . . . . . . . . . . . . . . . . . . . . . 998

3 The $\mathscr{C}=m$ Besicovitch-Federer Theorem . . . . . . . . . . . . . . . . . . . . . . . . 1001

3.1 Main theorem . . . . . . . . . . . . . . . . . . . . . . . . . 1001

3.2 Reverse implication . . . . . . . . . . . . . . . . . . . . . . . . . . . . 1007

3.3 Potential extension of the theorem . . . . . . . . . . . . . . . . . . . . . . . 1009

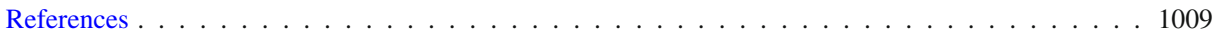

Jacek Gałęski

jg255456@mimuw.edu.pl

1 Institute of Mathematics, University of Warsaw, Banacha 2, Room 5040, 02-097 Warszawa, Poland 


\section{Introduction}

Rectifiability is one of the most important concepts investigated in geometric measure theory. A rectifiable set coupled with an orientation and integer multiplicity results in a notion of an integral current which has two nice properties. First, the space of such currents enjoys a compactness property proved by Federer and Fleming in [6], and second, it contains the class of smooth orientable surfaces, and thus constitutes its natural generalisation.

The subject of this work originates from a modification of a systematic way of projecting cubes of Whitney partition onto their $m$-dimensional skeletons, see [3, Chapter 3]. Let $\mathscr{H}^{m}$ denote the $m$-dimensional Hausdorff measure. In [1,2.9] Almgren constructs a similar smooth mapping $l_{m}$ from almost all of $\mathbb{R}^{n}$ to the $m$-dimensional skeleton of cubes. Then, in Section 2.9(b) on the page 338 of the same work, Almgren claims that for a purely $m$-unrectifiable set $\Sigma$ with $\mathscr{H}^{m}(\Sigma)<\infty$, there exists a perturbation $l_{m}^{*}$ arbitrarily close to $l_{m}$ in $\mathscr{C}^{1}$ topology such that $\mathscr{H}^{m}\left(l_{m}^{*}(\Sigma)\right)=0$. We provide a proof of this claim for "an arbitrary mapping having constant rank of the Jacobian matrix" instead of the map $l_{m}$.

The proof is based on a construction of a diffeomorphism $\Xi_{\varepsilon}$ such that $\mathscr{H}^{m}\left(f \circ \Xi_{\varepsilon}(\Sigma)\right)=0$. The significance of class $\mathscr{C}^{1}$ comes from the potential application. A map of class $\mathscr{C}^{1}$ allows for a continuous pushforward of integral currents. This may be a useful tool in proving the existence of solutions, in this class, to variational problems, for example the Plateau problem. In this particular problem removing the unrectifiable part by a slight perturbation strictly decreases the measure, so it seems like a step in the right direction.

A similar problem has been recently studied by Pugh in [10]. In this work Pugh investigates Lipschitz mappings arbitrarily close to identity in $\mathscr{C}^{0}$ topology such that the measure of the image under this mappings of an unrectifiable part is arbitrarily small. Moreover, if this unrectifiable part was a part of a larger set, the rectifiable part of the larger set is changed only slightly under this mapping. Note that Pugh constructs a Lipschitz map that alone abates the measure of unrectifiable part. We, on the other hand, build a diffeomorphism of the domain of a given map $f$ such that after a composition with $f$ the measure of the unrectifiable part is zero.

Furthermore the solution to the above problem left by Almgren in the case of radial projections can be found in thesis by [7, Lemme 4.3.16, p. 119].

Let $n, m \in \mathbb{N}$ be such that $m<n$. We say the set $E \subset \mathbb{R}^{n}$ is $m$-rectifiable if there exists a countable family of Lipschitz maps $f_{i}: \mathbb{R}^{m} \rightarrow \mathbb{R}^{n}$ for $i \in \mathbb{N}$, such that $\mathscr{H}^{m}\left(E \backslash \bigcup f_{i}\left(\mathbb{R}^{m}\right)\right)=0$. We say that the set $\Sigma$ is purely $m$-unrectifiable if $\mathscr{H}^{m}(\Sigma \cap E)=0$ for every $m$-rectifiable set $E$.

The Besicovitch-Federer projection theorem was first proved by Besicovitch in [2] for one dimensional sets on the plane and then generalised to any dimension by Federer in [4]. We use the formulation given in [9, Theorem 18.1] which consists of two statements. The first one characterises rectifiable sets: a set $A$ is $m$-rectifiable if and only if the image of every subset $B \subset A$ of positive $\mathscr{H}^{m}$-measure under a generic projection has positive $\mathscr{H}^{m}$-measure, i.e. $\mathscr{H}^{m}\left(P_{V}(B)\right)>0$. The second one concerns unrectifiable sets and states that for a generic orthogonal projection $P_{V}$ onto an $m$-dimensional plane $V$ the measure of the image of a purely $m$-unrectifiable set $\Sigma$ is zero, i.e. $\mathscr{H}^{m}\left(P_{V}(\Sigma)\right)=0$. The statement about rectifiable sets expresses the intuitions: on the plane, the shadow cast by an interval has positive length unless the interval is parallel to the light rays. Moreover, we see that that 1-unrectifiable sets despite having positive $\mathscr{H}^{1}$-measure cast shadow of length zero for almost all directions of light rays. 
On the pages that follow we generalise the second of the above statements. We consider the consequences of the substitution of the orthogonal projections in the B-F theorem by the class of mappings having constant rank of the Jacobian matrix. For an open set $U \subset \mathbb{R}^{n}$, we denote this class by

$$
\mathscr{C}_{=m}^{1}\left(U, \mathbb{R}^{n}\right):=\left\{f \in \mathscr{C}^{1}\left(U, \mathbb{R}^{n}\right) \mid \operatorname{dim} \operatorname{im} D f(x)=m, \forall x \in U\right\} .
$$

This is a much wider class of mappings, and consequently our assertion differs from the result of the theorem of Besicovitch and Federer. Instead of a measure theoretic result we obtain a topological one involving the density. Precisely, in Sect. 3 we prove the following theorem.

Theorem 3.1 For a purely m-unrectifiable set $\Sigma$ contained in an open set $U \subset \mathbb{R}^{n}$, such that $\mathscr{H}^{m}(\Sigma)<\infty$, let

$$
\mathscr{A}(\Sigma):=\left\{f \in \mathscr{C}^{1}\left(U, \mathbb{R}^{n}\right) \mid \operatorname{dim} \operatorname{im} D f=m, \mathscr{H}^{m}(f(\Sigma))>0\right\} .
$$

Then the interior of the set $\mathscr{A}(\Sigma)$ in the $\mathscr{C}_{=m}^{1}\left(U, \mathbb{R}^{n}\right)$ topology is empty.

The proof is based on a construction of a diffeomorphism of the domain that can be arbitrarily close to the identity, and such that the pullback of $f \in \mathscr{A}(\Sigma)$ by this diffeomorphism is no longer in the set $\mathscr{A}(\Sigma)$. This construction is divided into two main steps. First, in Sect. 2, we prove a local variant of this theorem.

The proof of the local variant is based on the fact that for a mapping $f \in \mathscr{C}_{=m}^{1}\left(U, \mathbb{R}^{n}\right)$ and a point $x \in U$ there exists a neighbourhood $V$ of $x$ and diffeomorphisms $\phi$ and $\psi$ of $\mathbb{R}^{n}$ such that $\left.f\right|_{V}=\left.\psi^{-1} \circ \pi \circ \phi\right|_{V}$, where $\pi$ is an orthogonal projection onto some $m$-plane. We insert a rotation $\theta$ of $\mathbb{R}^{n}$ into this composition to obtain

$$
f_{\varepsilon}=f \circ \Xi=\left(\psi^{-1} \circ \pi \circ \phi\right) \circ\left(\phi^{-1} \circ \theta \circ \phi\right) .
$$

We then show that $\theta$ can be chosen so that $f_{\varepsilon}$ is closer to $f$ than $\varepsilon$ in $\mathscr{C}^{1}$ topology and zeroes the measure of unrectifiable $\Sigma$ in the image, i.e. $\mathscr{H}^{m}\left(f_{\varepsilon}(\Sigma \cap V)\right)=0$.

Next, in Sect. 3 we prove the main theorem. The crucial difficulty is to glue diffeomorphisms $\Xi=\phi^{-1} \circ \theta \circ \phi$, that do not agree on intersections and act non-trivially on boundaries of their individual domains, constructed in the first step of the proof. This is achieved by a careful construction of a countable family $\mathscr{F}$ of open, connected, pairwise disjoint sets, whose closures covers the domain $U$. Then, we iteratively apply the modified diffeomorphisms constructed in the local variant of the theorem to elements of the family $\mathscr{F}$.

In order to obtain this modification we interpolate between diffeomorphism from the local variant and the identity using a vector flow. The result is that away from the boundary of an element of the family $\mathscr{F}$ the modified diffeomorphism is equal to the one from the local variant, and on the boundary it is equal to the identity. Once we have applied this modified diffeomorphism, the part of the set $\Sigma$ that was away from the boundary, after further composition with $f$, yields no measure.

It is not the case when we consider the part of the set $\Sigma$ that is near the boundary. On the collar around the boundary the modified diffeomorphism is not equal to the mapping from the local variant and consequently we do not know the outcoming measure of the perturbed $\Sigma$ after the composition with $f$. The solution is to iterate modified diffeomorphisms as described in previous paragraph on decreasingly thinner collars around the boundaries.

In Sect. 3.2 we describe the reverse implication i.e., if the set $\Sigma \subset U$ has positive measure and $\mathscr{A}(\Sigma)$ has empty interior in $\mathscr{C}_{=m}^{1}\left(U, \mathbb{R}^{n}\right)$ then $\Sigma$ is $m$-purely unrectifiable. Therefore the property in Theorem 3.1 is in fact equivalent description of purely unrectifiable sets. 
Instead of $\mathscr{C}_{=m}^{1}\left(U, \mathbb{R}^{n}\right)$ we may consider even a wider class of mappings having bounded rank of the Jacobian matrix, namely

$$
\mathscr{C}_{\leq m}^{1}\left(U, \mathbb{R}^{n}\right):=\left\{f \in \mathscr{C}^{1}\left(U, \mathbb{R}^{n}\right) \mid \operatorname{dim} \operatorname{im} D f(x) \leq m, \forall x \in U\right\} .
$$

In Sect. 3.3 we discuss the potential extension of the main theorem. The argument would be a simple consequence of Sard's theorem under the following conjecture.

Conjecture 1.1

$$
\mathscr{C}_{\leq m}^{\infty}\left(U, \mathbb{R}^{n}\right) \text { is dense in } \mathscr{C}_{\leq m}^{1}\left(U, \mathbb{R}^{n}\right) .
$$

The difficulty in proving this conjecture is that for $f \in \mathscr{C}_{\leq m}^{1}\left(U, \mathbb{R}^{n}\right)$ the convolution with the mollifier $\vartheta_{\varepsilon} \star f \notin \mathscr{C}_{\leq m}^{\infty}\left(U, \mathbb{R}^{n}\right)$ because rank of the Jacobian matrix is generally no longer bounded by $m$.

\subsection{Notation and conventions}

1. For $r \in \mathbb{R}$ and $A \subset \mathbb{R}^{n}$ the $r$-neighbourhood of the set $A$ is

$$
B_{r}(A):=\bigcup_{p \in A} B(p, r)
$$

2. The Standard mollifier $\vartheta$ is given by

$$
\vartheta(x):=\left\{\begin{array}{ll}
\exp \left(\frac{-1}{\left(1-|x|^{2}\right)}\right) / I_{n} & \text { for }|x|<1 \\
0 & \text { for }|x| \geq 1
\end{array},\right.
$$

where $I_{n}$ is such that $\int_{\mathbb{R}^{n}} \vartheta(x) d x=1$ and also denote

$$
\vartheta_{\varepsilon}(x):=\varepsilon^{-n} \vartheta\left(\varepsilon^{-1} x\right) \text {. }
$$

\section{Local variant}

Before we discuss the local variant of the theorem we shall explain how the orthogonal projections and the class $\mathscr{C}_{=m}^{1}\left(U, \mathbb{R}^{n}\right)$ are related to each other.

For a continuously differentiable map $f: \mathbb{R}^{n} \rightarrow \mathbb{R}^{m}$ with $r(D f):=\operatorname{dim} \operatorname{im}(D f)=m$ at some point $y$ there exists [11, Th. 2-13 p. 43] a neighbourhood $V \ni y$ and a mapping $h: V \rightarrow \mathbb{R}^{n}$ such that for $\left(x_{1}, \ldots, x_{n}\right) \in V$ we have

$$
f \circ h\left(x_{1}, x_{2}, \ldots, x_{n}\right)=\left(x_{n-m+1}, x_{n-m+2}, \ldots, x_{n}\right) .
$$

In other words we say: there exists a diffeomorphism that straightens preimages $f^{-1}(y)$ for a $y \in \mathbb{R}^{m}$. Its inverse is the diffeomorphism $h$ mentioned above.

The image of a mapping $f$ from $\mathscr{C}_{=m}^{k}\left(\mathbb{R}^{n}, \mathbb{R}^{n}\right)$ is locally an $m$-dimensional manifold, thus there exists a diffeomorphism $\psi: W \rightarrow \mathbb{R}^{n}$ of a neighbourhood $W \subset \mathbb{R}^{n}$ of the point $f(a)$ such that $\psi(f(U) \cap W)$ is contained in the plane $\left\{\left(x_{1}, \ldots, x_{n}\right) \in \mathbb{R}^{n} \mid x_{m+1}=x_{m+2}=\right.$ $\left.\cdots=x_{n}=0\right\}$. By combining this observation and Eq. (4) we obtain the classical result.

Corollary 2.1 (Constant rank theorem) Suppose $f \in \mathscr{C}_{=m}^{k}\left(U, \mathbb{R}^{n}\right)$. For any $x \in U$ there exist open sets $U_{x} \ni x$ and $W_{f(x)} \ni f(x)$, and diffeomorphisms $\phi_{x}: U_{x} \rightarrow \mathbb{R}^{n}$ and $\psi_{f(x)}: W_{f(x)} \rightarrow \mathbb{R}^{n}$ of class $\mathscr{C}^{k}$ such that the mapping $\left(\psi_{f(x)} \circ f \circ \phi_{x}{ }^{-1}\right): \phi_{x}\left(U_{x}\right) \rightarrow \mathbb{R}^{n}$ satisfies:

$$
\left(\psi_{f(x)} \circ f \circ \phi_{x}^{-1}\right)=\left.P_{V}\right|_{\phi_{x}\left(U_{x}\right)},
$$


where $P_{V}: \mathbb{R}^{n} \rightarrow \mathbb{R}^{n}$ is the projection on an m-dimensional plane $V$.

Therefore any map $f \in \mathscr{C}_{=m}^{k}\left(U, \mathbb{R}^{n}\right)$ is similar in the above local manner, to the orthogonal projection onto an $m$-dimensional plane. Therefore, the class $\mathscr{C}_{=m}^{1}\left(U, \mathbb{R}^{n}\right)$ is a natural generalisation of projections.

Our temporary goal is the following local form of the main theorem.

Theorem 2.2 (Local variant) Let $\varepsilon>0, \Sigma$ be an m-unrectifiable set, such that $\mathscr{H}^{m}(\Sigma)<\infty$ and $f \in \mathscr{C}_{=m}^{1}\left(U, \mathbb{R}^{n}\right)$. For any point $x \in U$ there exist a neighbourhood $U(x, r)$, and $f_{\varepsilon} \in \mathscr{C}_{=m}^{1}\left(U(x, r), \mathbb{R}^{n}\right)$ such that

$$
\left\|f-f_{\varepsilon}\right\|_{\mathscr{C}^{1}\left(U(x, r), \mathbb{R}^{n}\right)} \leq \varepsilon \text { and } \mathscr{H}^{m}\left(f_{\varepsilon}(\Sigma \cap U(x, r))\right)=0 .
$$

We precede the proof with a proposition stating that the unrectifiability property is preserved by a bi-Lipschitz diffeomorphism.

Proposition 2.3 Let $\Sigma \subset U$ be a purely m-unrectifiable set with $\mathscr{H}^{m}(\Sigma)<\infty$ and $\phi: U \rightarrow V$ be a bi-Lipschitz diffeomorphism. Then $\phi(\Sigma)$ is also an m-unrectifiable set.

Proof The $m$-dimensional Hausdorff measure satisfies the inequality (see [9, Theorem 7.5, p. 103])

$$
\mathscr{H}^{m}(l(A)) \leq \operatorname{Lip}(l)^{m} \mathscr{H}^{m}(A),
$$

where $l: \mathbb{R}^{n} \rightarrow \mathbb{R}^{n}$ is a Lipschitz map and $A$ is a subset of $\mathbb{R}^{n}$. Therefore, for any $m$-rectifiable set $E \subset V$ we see that

$$
\mathscr{H}^{m}(\phi(\Sigma) \cap E) \leq \operatorname{Lip}\left(\phi^{-1}\right)^{m} \mathscr{H}^{m}\left(\Sigma \cap \phi^{-1}(E)\right)=0,
$$

where the last equality follows from the rectifiability of $\phi^{-1}(E)$.

We are now in a good position to prove the lemma.

Proof of Theorem 2.2 We divide the proof into three parts. The first part contains the definition of the diffeomorphism $\Xi_{\theta}$ which composed with map $f$ will be the mapping $f_{\varepsilon}=f \circ \Xi_{\theta}$ from the conclusion of the theorem. In the second part we investigate the distance between $f$ and the modified map. In the last part we check when the image $f \circ \Xi_{\theta}(\Sigma)$ has $m$-dimensional measure equal to zero.

Step 1 Let $U_{x}$ be an open neighbourhood as in Theorem 2.1. Using a translation we can assume that $0=\phi_{x}(x)$. For every point $x \in U$ let $r_{x}=\operatorname{dist}\left(0, \partial \phi_{x}\left(U_{x}\right)\right)$. Then for any positive $r<r_{x}$ we have $\bar{B}(0, r) \subset \subset \phi_{x}\left(U_{x}\right)$. For any such $r$ we define

$$
U(x, r):=\phi_{x}^{-1}(B(0, r)) \subset U_{x} .
$$

For simplicity denote $\phi:=\phi_{x}$. For an isometry $S O(n) \ni \theta: \mathbb{R}^{n} \rightarrow \mathbb{R}^{n}$ let $\Xi_{\theta}$ stand for the map

$$
\Xi_{\theta}=\phi^{-1} \circ \theta \circ \phi: U(x, r) \rightarrow U(x, r) .
$$

Observe that the further composition with $(\psi \circ f)$ yields a mapping from $U(x, r)$ to $\mathbb{R}^{m}$. We investigate the image of the set $\Sigma$ under this composition

$$
\begin{aligned}
\psi \circ f \circ \Xi_{\theta}(\Sigma) & =\left(\psi \circ f \circ \phi^{-1}\right) \circ \theta \circ \phi(\Sigma) \\
& =\left(P_{V} \circ \theta\right)(\phi(\Sigma)),
\end{aligned}
$$


for some $m$-dimensional plane $V$. We claim that $P_{V} \circ \theta=\theta \circ P_{\theta^{-1}(V)}$. Indeed, by computing the preimage of a point $v \in V$, we have

$$
\begin{aligned}
\left(P_{V} \circ \theta\right)^{-1}(v) & =\theta^{-1}\left(P_{V}^{-1}(v)\right) \\
& =\theta^{-1}\left(v+V^{\perp}\right) \\
& =\theta^{-1}(v)+\theta^{-1}\left(V^{\perp}\right),
\end{aligned}
$$

while

$$
\begin{aligned}
\left(\theta \circ P_{\theta^{-1}(V)}\right)^{-1}(v) & =\left(P_{\theta^{-1}(V)}\right)^{-1}\left(\theta^{-1}(v)\right) \\
& =\theta^{-1}(v)+\theta^{-1}(V)^{\perp} \\
& =\theta^{-1}(v)+\theta^{-1}\left(V^{\perp}\right) .
\end{aligned}
$$

Therefore, we obtained a projection of the unrectifiable set $\phi(\Sigma)$ onto a subspace $\theta^{-1}(V)$. We will choose symmetry $\theta$ is such a way that it is both close to identity mapping and the projection onto the $m$-plane $\theta^{-1}(V)$ zeroes the measure of $\phi(\Sigma)$.

Step 2 We will prove that there exists an open neighbourhood of id in $S O(n)$ such that the composition

$$
f \circ \Xi_{\theta}: U(x, r) \rightarrow \mathbb{R}^{n}
$$

can be as close to the mapping $\left.f\right|_{U(x, r)}$ as we wish. Observe that both $f$ and $D f$ are continuous up to the boundary of $U(x, r)$ for $r<r_{x}$, hence, they are uniformly continuous. For the differential $D f$ define the continuity parameters $\tilde{\varepsilon}$ and $\tilde{\delta}$ by the following statement: for any positive $\tilde{\varepsilon}$ there exists $\tilde{\delta}=\tilde{\delta}(\tilde{\varepsilon})$, such that if $|y-x| \leq \tilde{\delta}$, then $\|D f(y)-D f(x)\| \leq \tilde{\varepsilon}$. We estimate

$$
\begin{aligned}
\left\|f \circ \Xi_{\theta}-f\right\|_{\mathscr{C}^{1}}= & \left\|f \circ \Xi_{\theta}-f\right\|_{\mathscr{C}^{0}}+\left\|D f\left(\Xi_{\theta}\right) \cdot D \Xi_{\theta}-D f\right\|_{\mathscr{C}^{0}} \\
\leq & \|f\|_{\mathscr{C}^{1}}\left\|\Xi_{\theta}-\mathrm{id}\right\|_{\mathscr{C}^{0}}+\left\|D f\left(\Xi_{\theta}\right) \cdot\left(D \Xi_{\theta}-\mathrm{id}+\mathrm{id}\right)-D f\right\|_{\mathscr{C}^{0}} \\
\leq & \|f\|_{\mathscr{C}^{1}}\left\|\Xi_{\theta}-\mathrm{id}\right\|_{\mathscr{C}^{0}}+\left\|D f\left(\Xi_{\theta}\right) \cdot\left(D \Xi_{\theta}-\mathrm{id}\right)\right\|_{\mathscr{C}^{0}} \\
& +\left\|D f\left(\Xi_{\theta}\right)-D f\right\|_{\mathscr{C}^{0}} \\
\leq & \|f\|_{\mathscr{C}^{1}}\left\|\Xi_{\theta}-\mathrm{id}\right\|_{\mathscr{C}^{0}}+\|D f\|_{\mathscr{C}^{0}}\left\|\left(D \Xi_{\theta}-\mathrm{id}\right)\right\|_{\mathscr{C}^{0}} \\
& +\left\|D f\left(\Xi_{\theta}\right)-D f\right\|_{\mathscr{C}^{0}} \\
\leq & C\left(\|f\|_{\mathscr{C}^{1}}\right)\left\|\Xi_{\theta}-\mathrm{id}\right\|_{\mathscr{C}^{1}}+\tilde{\varepsilon}
\end{aligned}
$$

for $\left\|\Xi_{\theta}-\mathrm{id}\right\|_{\mathscr{C}^{0}}<\tilde{\delta}(\tilde{\varepsilon})$. Observe, that if we pick $\tilde{\varepsilon}<\varepsilon / 2$ and $\left\|\Xi_{\theta}-\mathrm{id}\right\|_{\mathscr{C}^{0}}<\min (\tilde{\delta}, \varepsilon / 2)$ then the left hand side of Eq. (12) is less than $\varepsilon$. Therefore, we need to control the $\mathscr{C}^{1}$ norm of $\left(\Xi_{\theta}-\mathrm{id}\right)$. Similarly to Eq. (12) one can show that also $\left\|\Xi_{\theta}-\mathrm{id}\right\|_{\mathscr{C}}{ }^{1}(U(x, r))$ is continuous in the argument $\theta$. This provides that for any positive $\varepsilon$ we can find an open ball in $S O(n)$

$$
B_{S O}(\mathrm{id}, \rho):=\left\{\theta \mid\|\theta-\mathrm{id}\|_{\mathscr{C} 1}<\rho\right\},
$$

such that

$$
\left\|f \circ \Xi_{\theta}-\mathrm{id}\right\|_{\mathscr{C} 1}<\varepsilon \text { for } \theta \in B_{S O}(\mathrm{id}, \rho) \text {. }
$$

Remark 2.4 Let $V$ be an $m$-plane in $\mathbb{R}^{n}$ which is an element of the Grassmann manifold $\operatorname{Gr}(n, m)$. Then

$$
B_{S O}(\mathrm{id}, \rho) . V=\left\{\theta(V) \in G r(n, m) \mid \theta \in B_{S O}(\mathrm{id}, \rho)\right\}
$$


is a set of positive $\gamma_{n, m}$-measure. Indeed, let $v$ be the uniformly distributed measure on the orthogonal group $O(n)$. From the definition of $\gamma_{n, m}$ we have

$$
\begin{aligned}
\gamma_{n, m}\left(B_{S O}(\mathrm{id}, \rho) . V\right) & \stackrel{\text { def }}{=} v\left(\left\{g \in O(n) \mid g . V \in B_{S O}(\mathrm{id}, \rho) . V\right\}\right) \\
& =v\left(B_{S O}(\mathrm{id}, \rho)\right)>0,
\end{aligned}
$$

because $B_{S O}$ (id, $\rho$ ) is an open set also in $O(n)$.

Step 3 From Besicovitch-Federer theorem [9, Theorem 18.1] in the open set $B_{S O}$ (id, $\left.\rho\right) . V$ define the set $Z_{\phi(\Sigma)}$ consisting of those $m$-planes for which

$$
\mathscr{H}^{m}\left(P_{\theta^{-1}(V)}(\phi(\Sigma))\right)=0 \text { for } \theta^{-1}(V) \in Z_{\phi(\Sigma)}
$$

and then $\gamma_{n, m}\left(B_{S O}(\right.$ id, $\left.\rho) . V\right)=\gamma_{n, m}\left(Z_{\phi(\Sigma)}\right)$. Under the mappings $\psi^{-1}$ and $\theta$ a set of zero $\mathscr{H}^{m}$-measure is mapped to a set of zero measure, hence we have

$$
\begin{aligned}
0=\mathscr{H}^{m}\left(P_{\theta^{-1}(V)}(\phi(\Sigma))\right) & =\mathscr{H}^{m}\left(\theta \circ P_{\theta^{-1}(V)}(\phi(\Sigma))\right) \\
\text { by Eq. }(8) & =\mathscr{H}^{m}\left(\psi \circ f \circ \phi^{-1} \circ \theta \circ \phi(\Sigma)\right) \\
& =\mathscr{H}^{m}\left(f \circ \Xi_{\theta}(\Sigma)\right) .
\end{aligned}
$$

\section{The $\mathscr{C}_{=m}^{\mathbf{1}}$ Besicovitch-Federer Theorem}

In this section we prove the main theorem and the reverse implication, thus we obtain a characterisation of purely unrectifiable sets $\Sigma$ in terms of the topology of $\mathscr{A}(\Sigma)$.

\subsection{Main theorem}

The main theorem states, in slightly other words, that for any $\mathscr{C}^{1}$ neighbourhood of a function $f \in \mathscr{C}_{=m}^{1}\left(U, \mathbb{R}^{n}\right)$ there exists perturbation $f_{\varepsilon}$ such that an $f_{\varepsilon}(\Sigma)$ has zero measure.

Theorem 3.1 For a purely m-unrectifiable set $\Sigma$ contained in an open set $U \subset \mathbb{R}^{n}$, such that $\mathscr{H}^{m}(\Sigma)<\infty$, let

$$
\mathscr{A}(\Sigma):=\left\{f \in \mathscr{C}^{1}\left(U, \mathbb{R}^{n}\right) \mid \operatorname{dim} \operatorname{im} D f=m, \mathscr{H}^{m}(f(\Sigma))>0\right\} .
$$

Then the interior of the set $\mathscr{A}(\Sigma)$ in the $\mathscr{C}_{=m}^{1}\left(U, \mathbb{R}^{n}\right)$ topology is empty.

Proof We will prove that for every $\varepsilon>0$ and $f \in \mathscr{A}(\Sigma)$ there exists $f_{\varepsilon} \in \mathscr{C}_{=m}^{1}$ such that $f_{\varepsilon} \notin \mathscr{A}(\Sigma)$ and $\left\|f-f_{\varepsilon}\right\|_{\mathscr{C}^{1}(U)} \leq \varepsilon$, which will imply that the interior of $\mathscr{A}(\Sigma)$ is empty. The function $f_{\varepsilon}$ will be the limit of a sequence $f_{n}$ contained in an $\varepsilon$-neighbourhood of $f$ and such that $\mathscr{H}^{n}\left(f_{n}(\Sigma)\right) \stackrel{n \rightarrow \infty}{\longrightarrow} 0$.

From Theorem 2.2 we already know that locally Theorem 3.1 is true. The problem is that the diffeomorphisms $\Xi_{\theta}$ do not agree on overlapping sets $U(x, r)$ and $U(\tilde{x}, \tilde{r})$. Thus we are forced to work on disjoint sets. Additionally diffeomorphisms $\Xi_{\theta}$ acts non-trivially on the boundary of $U(x, r)$, hence those sets have to be not only disjoint but also have positive distance between each other.

The following lemma enables us to extend a mapping $\Xi_{\theta}$ from the Theorem 2.2 to a larger set. For a subset $\mathscr{O}$ contained in $U(x, r)$, provided that is is far enough from the boundary of $U(x, r)$, we will find a diffeomorphism which acts like $\Xi_{\theta}$ on $\mathscr{O}$ and equals to identity outside some neighbourhood of the set $\mathscr{O}$. 
Lemma 3.2 If a compact set $U$ is $\mathscr{C}^{1}$-diffeomorphic to a closed ball through some $\varphi: \bar{U} \rightarrow$ $\bar{B}(0, r)$ and

(a) $\mathscr{O} \subset U \backslash B_{\mu}(\partial U)$, for some $\mu>0$;

(b) $\eta>0$,

then there exists $\rho>0$ such that for any $\theta \in B_{S O}(i d, \rho) \backslash\{i d\}$ there exists a diffeomorphism

$$
\zeta:=\zeta(\mu, \eta, \theta): U \rightarrow U
$$

with the following properties:

(i) $\zeta\left(B_{\mu / 4}(\mathscr{O})\right) \subseteq B_{\mu / 2}(\mathscr{O})$ and $\zeta(y)=\Xi_{\theta}(y)$ for $y \in B_{\mu / 4}(\mathscr{O})$;

(ii) $\zeta(y)=y$ for $y \in U \backslash B_{3 \mu / 4}(\mathscr{O})$;

(iii) $\|\zeta-i d\|_{\mathscr{C}^{1}(U)} \leq \eta$.

Proof For an element $\theta \in S O(n)$ sufficiently close to id (before cut locus) there exists a unique path

$$
\exp \left(t X_{\theta}\right)=\theta_{t}: \mathbb{R} \rightarrow S O(n) \text { for } X_{\theta} \in \mathfrak{s o}(n)
$$

connecting id and $\theta$ that is $\theta_{1}=\theta, \theta_{0}=\mathrm{id}$. Mapping $t \mapsto \exp \left(t X_{\theta}\right)$ is a group endomorphism $\mathbb{R} \rightarrow \operatorname{Diff}(B(0, r))$, in other words it is a flow, and induces a vector field

$$
X(b)=\left.\frac{d}{d t} \exp \left(t X_{\theta}\right)(b)\right|_{t=0} \text { for } b \in B(0, r) .
$$

The aim now is to produce a vector field $V_{\theta} \in \Gamma(T U)$ such that its vector flow gives the mapping $\Xi_{\theta}(y)=\varphi_{V_{\theta}}(1, y)$. Notice that the field $X$ can be transported back to the set $U$ via diffeomorphism $\phi$ or again using the fact that the flow $\Xi_{\theta_{t}}: \mathbb{R} \times U \rightarrow U$ generates a vector field

$$
\begin{aligned}
V_{\theta}(y):=\left.\frac{d}{d t} \Xi_{\theta_{t}}(y)\right|_{t=0} & =\left.\frac{d}{d t} \phi^{-1} \circ \theta_{t} \circ \phi(y)\right|_{t=0} \\
& =\left.D \phi^{-1}\left(\theta_{0} \circ \phi(y)\right) \frac{d}{d t} \theta_{t}(\phi(y))\right|_{t=0} \\
& =D \phi^{-1}(\phi(y)) . X(\phi(y)) \\
& =\phi^{-1}{ }_{*}\left(\phi^{*} X\right)(y) \quad \text { for } y \in U .
\end{aligned}
$$

Multiplying the field $V_{\theta}$ by a smooth cutoff function of an appropriate neighbourhood of the set $\mathscr{O}$ we obtain a flow that at small enough time will do exactly what was formulated in the points (i)-(iii).

Let

$$
W_{\theta}(y):=\left(\vartheta_{\mu / 8} * \mathbb{1}_{B_{5 \mu / 8}(\mathscr{O})}\right)(y) \cdot V_{\theta}(y) .
$$

By $\varphi_{W \theta}(t, y)$ we denote the trajectory of the point $y$ at the time $t$ under the flow of the field $W_{\theta}$. Since for every point $y$ in $\mu / 2$-neighbourhood of the set $\mathscr{O}$, denoted by $B_{\mu / 2}(\mathscr{O})$, the support of the mollifier $\vartheta_{\mu / 8}(\cdot-y)$ is contained in $(\mu / 2+\mu / 8)$-neighbourhood of $\mathscr{O}$, the field $W_{\theta}$ is equal to $V_{\theta}$ on $B_{\mu / 2}(\mathscr{O})$. Therefore, there exists $t$ such that the flow $\varphi_{W_{\theta}}(s, y)=\Xi_{\theta_{s}}(y)$ for $y \in B_{\mu / 4}(\mathscr{O})$ and $s \leq t$. This gives the proof of the point (i).

The field $W_{\theta}$ is equal to zero outside $\operatorname{spt}\left(\vartheta_{\mu / 8} * \mathbb{1}_{B_{5 \mu / 8}(\mathscr{O})}\right) \subseteq B_{3 \mu / 4}(\mathscr{O})$, hence for $y \in$ $B_{3 \mu / 4}(\mathscr{O})^{c}$ we have $\varphi_{W}(t, y)=y$ and the point (ii).

The field $W_{\theta}$ is Lipschitz on the compact set $\bar{U}$ and equal to zero on the neighbourhood of the boundary, and generates one-parameter group of diffeomorphisms that exists for times 
$t \in(-\varepsilon, \varepsilon)$ for some positive $\varepsilon$. Differentiable dependence on initial conditions guaranties that we will find time $t$ so small that item (iii) is satisfied for all $|s|<t$.

So far for some fixed $\theta$ we found $t$ such that $\theta_{t}$ fulfils (i), (ii) and (iii). Observe that the mapping

$$
\begin{aligned}
S O(n) & \longrightarrow \operatorname{Diff}_{\mathscr{C}^{1}}(U) \\
\theta & \mapsto \varphi_{W_{\theta}}(1, \cdot)
\end{aligned}
$$

is continuous, hence $\theta \mapsto\left\|\varphi_{W_{\theta}}(1, \cdot)-\mathrm{id}\right\|_{\mathscr{C} 1}$ is also continuous. Therefore, there exists an open neighbourhood of id such that properties (i), (ii) and (iii) are satisfied.

Outline of the proof of theorem 3.1. First we shall construct a countable family $\mathfrak{U}$ of pairwise disjoint connected open sets $\left\{U_{i}\right\}$, such that every $U_{i}$ is contained in some domain $U(x, r)=$ $U(x(i), r(i))$. The set $U$ will be covered by the sum of closures of $U_{i}$ such that the measure $\mathscr{H}^{m}\left(\Sigma \cap \partial U_{i}\right)=0$ for every $i$.

Consider an element $U_{1}$ of the family $\mathfrak{U}$. By Lemma 3.2 there exists a diffeomorphism $\zeta_{1,1}$ such that on the set $\mathscr{O}:=U_{1} \backslash B_{\mu}\left(\partial U_{1}\right)$ it is equal to $\Xi_{\theta}$. The map $\Xi_{\theta}$ is chosen, using Theorem 2.2, in such a way that the $m$-measure of the image of $\mathscr{O} \cap \Sigma$ under $f \circ \zeta_{1,1}$ is zero, i.e. $\mathscr{H}^{m}\left(f \circ \Xi_{\theta}(\mathscr{O} \cap \Sigma)\right)=0$.

In the set $\zeta_{1,1}\left(U_{1}\right)$ the only possible place for positive $\mathscr{H}^{m}$ measure of $f \circ \zeta_{1,1}(\Sigma)$ is outside $\zeta_{1,1}(\mathscr{O})$. Define this set by $U_{1,2}:=U_{1} \backslash \zeta_{1,1}(\mathscr{O})$. The idea is to repeat the above procedure: apply Lemma 3.2 to the set $U_{1,2} \backslash B_{\mu_{2}}\left(\partial U_{1,2}\right)$. This yields a diffeomorphism $\zeta_{1,2}$ which slightly moves the set that is in $U_{1,2}$ and further than $\mu_{2}$ away from the boundary of $U_{1,2}$.

The sequence $f_{n}$ appears as the composition

$$
\begin{aligned}
f_{1} & =f \circ \zeta_{1,1} \\
f_{2} & =f \circ \zeta_{1,2} \circ \zeta_{1,1} \\
& \vdots \\
f_{n} & =f \circ \zeta_{1, n} \circ \ldots \circ \zeta_{1,1} .
\end{aligned}
$$

It will turn out that this sequence is a finite composition for almost all points $u \in U_{1}$. We have to check that this sequence is converging to some map $f_{\varepsilon}$. Here we end the outline and start the proof with a construction of the cover $\mathfrak{U}$.

Step 1. Construction of the family $\mathfrak{U}$ From now on let the family

$$
\widetilde{\mathfrak{B}} \subset\{U(x, r)\}_{x \in U, 0<r<r_{x}}
$$

stand only for those sets introduced in Theorem 2.2 that fulfil the property $\mathscr{H}^{m}(\Sigma \cap$ $\partial U(x, r))=0$. Note that for every $x$ in $U$ at most countable set of radii $r \in\left(0, r_{x}\right)$ does not have the above property. From this family choose a countable collection $\mathfrak{B} \subset \widetilde{\mathfrak{B}}$, covering $U$ and denote them $\mathfrak{B}=\left(V_{i}\right)_{i \in \mathbb{N}}$. Consider the following collection of open sets

$$
\widetilde{\mathfrak{U}}=\left\{\operatorname{int}\left(V_{1}\right), \operatorname{int}\left(V_{2}\right) \backslash \overline{V_{1}}, \operatorname{int}\left(V_{3}\right) \backslash \overline{\left(V_{1} \cup V_{2}\right)}, \ldots, \operatorname{int}\left(V_{n}\right) \backslash \overline{\bigcup_{i<n} V_{i}}, \ldots\right\} .
$$

Finally define $\mathfrak{U}$ as the family of connected components of the elements of $\widetilde{\mathfrak{U}}$. Denote the elements of $\mathfrak{U}$ by $\left(U_{i}\right)_{i \in \mathbb{N}}$. 
Example 3.3 On the plane, let $V_{1}=B(0,1), V_{2}=(-2,2) \times(-1,1)$ and $\mathfrak{B}=\left\{V_{1}, V_{2}\right\}$. In this case $\widetilde{\mathfrak{U}}$ consists of two sets: the ball $B(0,1)$ and the rectangle $(-1,1) \times(-1,1)$ with ball cut out. Then family $\mathfrak{U}$ has three elements: the ball, left remains from rectangle after cutting out the ball and symmetric right part.

Already $\operatorname{int}\left(V_{2}\right) \backslash \overline{V_{1}}$ can consist of countably many connected components. The support of the measure $\mathscr{H}^{m}\llcorner\Sigma$ is contained in sum of sets from $\mathfrak{U}$ because of the condition imposed on Eq. (17). Since

$$
\forall_{j \in \mathbb{N}} \exists_{n \in \mathbb{N}} \quad U_{j} \stackrel{\operatorname{conn}}{\complement} \operatorname{int}\left(V_{n}\right) \backslash \bigcup_{i<n} V_{i},
$$

the boundary of $U_{i}$ can be divided into finite sum

$$
\forall_{j \in \mathbb{N}} \exists_{n \in \mathbb{N}} \quad \partial U_{j} \subset \partial V_{n} \cup \partial V_{n-1} \cup \cdots \cup \partial V_{1} .
$$

Note that the $\partial U_{j} \cap \partial V_{i}$ can have infinitely many connected components.

Step 2. Controlling the $\mathscr{H}^{m}\llcorner\Sigma$-measure of the collars Observe that for a continuously differentiable, compact $(n-1)$ dimensional manifold $\partial V_{k}$ as above the limit

$$
\mathscr{H}^{m}\left(\Sigma \cap B_{\mu}\left(\partial V_{k}\right)\right) \longrightarrow 0 \text { as } \mu \rightarrow 0 \text {. }
$$

By contradiction, assume that $\lim _{\mu \rightarrow 0} \mathscr{H}^{m}\left(\Sigma \cap B_{\mu}\left(\partial V_{k}\right)\right)>0$. Then since $\Sigma$ has finite measure we have

$$
\mathscr{H}^{m}\left(\Sigma \cap \partial V_{k}\right)=\mathscr{H}^{m}\left(\Sigma \cap \bigcap_{\mu>0} B_{\mu}\left(\partial V_{k}\right)\right)=\lim _{\mu \rightarrow 0} \mathscr{H}^{m}\left(\Sigma \cap B_{\mu}\left(\partial V_{k}\right)\right)>0
$$

which contradicts the assumption about the family (17).

Fix $U_{i} \in \mathfrak{U}$ and let

$$
\sigma_{i}=\mathscr{H}^{m}\left(\Sigma \cap U_{i}\right) .
$$

Since $\partial U_{i} \subset \bigcup_{k<n} \partial V_{k}$ for some $n=n(i)$ then

$$
\mathscr{H}^{m}\left\llcorner\Sigma\left(B_{\mu}\left(\partial U_{i}\right)\right) \leq \sum_{k=1}^{n} \mathscr{H}^{m}\left\llcorner\Sigma\left(B_{\mu}\left(\partial V_{k}\right)\right) \longrightarrow 0 \text { as } \mu \rightarrow 0 .\right.\right.
$$

Therefore there exists $\mu_{i, 1}>0$ such that

$$
\mathscr{H}^{m}\left\llcorner( U _ { i } \cap \Sigma ) ( B _ { \mu _ { i , 1 } } ( \partial U _ { i } ) ) \leq \sigma _ { i } / 3 \text { and } \mathscr { H } ^ { m } \left\llcorner\left(U_{i} \cap \Sigma\right)\left(\partial\left(B_{\mu_{i, 1}}\left(\partial U_{i}\right)\right)\right)=0\right.\right. \text {. }
$$

We have second assertion because $\left\{\partial B_{s}\left(\partial U_{i}\right)\right\}_{s \in[0,1]}$ is an uncountable, pairwise disjoint family of sets which are $(n-1)$-rectifiable for almost all $s$.

Step 3. Using Lemma 3.2 We need to control the $\mathscr{H}^{m}\llcorner\Sigma$-measure in the collars around the boundary, because there we do not have property $\mathscr{H}^{m}(f \circ \zeta(\Sigma))=0$. Since $f$ is $\mathscr{C}^{1}$ up to the boundary of $V_{i}$, without loss of generality, we can assume that $\operatorname{Lip}(f) \leq 1$ and bound the measure of $\Sigma$ in the domain instead of the measure of the image. Let

$$
\varepsilon=\sum_{k=1}^{\infty} \varepsilon_{k} \quad \text { with } \varepsilon_{k}>0
$$

Apply Lemma 3.2 to the set $\mathscr{O}_{i}=U_{i} \backslash B_{\mu_{i, 1}}\left(\partial U_{i}\right)$ for fixed $U_{i} \in \mathfrak{U}$, with a positive

$$
\eta<\varepsilon_{1} / 3
$$


and $t$ in the vector flow $\varphi_{W_{\theta}}(t, \cdot)=\zeta$ so small that

$$
\mathscr{H}^{m}\left\llcorner\zeta(\Sigma)\left(\zeta\left(U_{i} \cap B_{\mu_{i, 1}}\left(\partial U_{i}\right)\right)\right) \leq \sigma_{i} / 2 .\right.
$$

Note that last step may increase the measure of $\zeta(\Sigma)$ in the deformed collar around boundary of the set $U_{i}$ compared to Eq. (23). Due to the continuity of function $t \mapsto \mathscr{H}^{m}\left(\varphi_{W_{\theta}}(t, \Sigma)\right)$, which follows from Eq. (5), it is possible to bound this increase by $\sigma_{i} / 2$. In Lemma 3.2 we deform a set $U$ diffeomorphic to some ball, here we know that $U_{i}$ is contained in some set $V_{j}$ which is diffeomorphic to a ball, see Eq. (17). Due to Theorem 2.2 we can choose a map $\theta$ such that $\zeta$ is equal to $\Xi_{\theta}$ on the set $\mathscr{O}_{i}$ and such that

$$
\mathscr{H}^{m}\left(f \circ \zeta\left(\Sigma \cap \mathscr{O}_{i}\right)\right)=0 .
$$

As a result the measure of $\Sigma$ vanishes in the set that is away from boundary of $U_{i}$.

Step 4. The iteration Now we will iterate the above construction. We are working with one element of the family $\mathfrak{U}$. Denote $U_{1}:=U_{i}, \mathscr{O}_{1}:=\mathscr{O}_{i}, \mu_{1}:=\mu_{i, 1}$ and also denote by $\zeta_{1}$ mapping $\zeta$ obtained in the previous point. Let

$$
\begin{aligned}
U_{2} & =U_{1} \cap \zeta_{1}\left(B_{\mu_{1}}\left(\partial U_{1}\right)\right) \\
\text { or shortly } & =U_{1} \backslash \zeta_{1}\left(\mathscr{O}_{1}\right) .
\end{aligned}
$$

This is the only possible place where, after composition with $f$, set $\zeta_{1}(\Sigma)$ yields positive measure. In the previous step the boundary of $U_{1}$ was $(n-1)$-rectifiable and we clearly had Eq. (22). Notice that the set changed, we are working now with $\zeta_{1}(\Sigma)$ inside a little collar around boundary of $U_{1}$ distorted by $\zeta_{1}$. Again we use fact that the boundary of $B_{\mu}(A)$ is rectifiable for any $A$ and almost all $\mu$. Hence we can choose $\mu_{2}$ such that

$$
\begin{gathered}
\mathscr{H}^{m}\left\llcorner\left(U_{2} \cap \zeta_{1}(\Sigma)\right)\left(\partial\left(B_{\mu_{2}}\left(\partial U_{2}\right)\right)\right)=0\right. \text { and } \\
\mathscr{H}^{m}\left\llcorner\left(U_{2} \cap \zeta_{1}(\Sigma)\right)\left(B_{\mu_{2}}\left(\partial U_{2}\right)\right) \leq \sigma / 3^{2} .\right.
\end{gathered}
$$

Apply Lemma 3.2 to the set $\mathscr{O}_{2}=U_{2} \backslash B_{\mu_{2}}\left(\partial U_{2}\right)$ with $\eta<\varepsilon_{2} / 3$ and $t$ in the flow such that

$$
\mathscr{H}^{m}\left(\zeta_{2}\left(\zeta_{1}(\Sigma) \cap U_{2} \cap B_{\mu_{2}}\left(\partial U_{2}\right)\right)\right)=\mathscr{H}^{m}\left(\zeta_{2}\left(\zeta_{1}(\Sigma) \cap U_{2} \backslash \mathscr{O}_{2}\right)\right) \leq \sigma / 2^{2} .
$$

We obtain the map $f_{2}=f \circ \zeta_{2} \circ \zeta_{1}$ with $\mathscr{H}^{m}\left(f_{2}\left(\Sigma \cap U_{1}\right)\right) \leq \sigma / 2^{2}$ as we assumed $\operatorname{Lip}(f) \leq 1$. Moreover

$$
\begin{aligned}
\left\|\zeta_{2} \circ \zeta_{1}-\mathrm{id}\right\|_{\mathscr{C}^{1}\left(U_{1}\right)} & =\left\|\zeta_{2} \circ \zeta_{1}-\zeta_{1}+\zeta_{1}-\mathrm{id}\right\|_{\mathscr{C}^{1}} \\
& \leq\left\|\zeta_{2} \circ \zeta_{1}-\zeta_{1}\right\|_{\mathscr{C}^{1}}+\left\|\zeta_{1}-\mathrm{id}\right\|_{\mathscr{C}^{1}} \\
& \leq \varepsilon_{2}+\varepsilon_{1} .
\end{aligned}
$$

If $f, g: U \rightarrow U$ are two diffeomorphisms $\varepsilon_{f}$ and $\varepsilon_{g}$ close to identity in $\mathscr{C}^{1}$ respectively, then

$$
\begin{aligned}
\|f \circ g-g\|_{\mathscr{C}^{1}} & =\|f \circ g-g\|_{\mathscr{C}^{0}}+\|D(f \circ g)-D(g)\|_{\mathscr{C}^{0}} \\
& =\|f-\mathrm{id}\|_{\mathscr{C}^{0}}+\|D f(g) \cdot D g-D(g)\|_{\mathscr{C}^{0}} \\
& \leq \varepsilon_{f}+\|D f(g)-\mathrm{id}\|_{\mathscr{C}^{0}}\|D g\|_{\mathscr{C}^{0}} \text { for }\|D g\| \in 1 \pm \varepsilon_{g} \\
& \leq \varepsilon_{f}+\varepsilon_{f}\left(1+\varepsilon_{g}\right)
\end{aligned}
$$

and if one take $\varepsilon_{f}<\varepsilon / 3$ and $\varepsilon_{g}<1 / 3$ then $\|f \circ g-g\|_{\mathscr{C}^{1}}<\varepsilon$. This is the reason for Eq. (25). 
Step 5. The $n$ th-step First we define $U_{n}=U_{n-1} \backslash \zeta_{n-1}\left(\mathscr{O}_{n-1}\right)$. Take a collar

$$
\operatorname{col}_{\mu_{n}}\left(U_{n}\right)=U_{n} \cap B_{\mu_{n}}\left(\partial U_{n}\right)
$$

around boundary of $U_{n}$ of width $\mu_{n}$. Since the boundary of $U_{n}$ and the boundary of the collar are rectifiable sets for almost all $\mu_{n}$, we can take $\mu_{n}$ such that

$$
\begin{gathered}
\mathscr{H}^{m}\left\llcorner\left(\zeta_{n-1} \circ \ldots \circ \zeta_{1}(\Sigma)\right)\left(\partial \operatorname{col}_{\mu_{n}}\left(U_{n}\right)\right)=0\right. \text { and } \\
\mathscr{H}^{m}\left\llcorner\left(\zeta_{n-1} \circ \ldots \circ \zeta_{1}(\Sigma)\right)\left(\operatorname{col}_{\mu_{n}}\left(U_{n}\right)\right) \leq \sigma / 3^{n} .\right.
\end{gathered}
$$

Use Lemma 3.2 to the set $\mathscr{O}_{n}=U_{n} \backslash \operatorname{col}_{\mu_{n}}\left(U_{n}\right)$ with $\eta<\varepsilon_{n} / 3$ and $t$ in the flow so small that

$$
\mathscr{H}^{m}\left(\zeta_{n}\left(\zeta_{n-1} \circ \ldots \circ \zeta_{1}(\Sigma) \cap \operatorname{col}_{n}\left(U_{n}\right)\right)\right) \leq \sigma / 2^{n}
$$

We estimate the distance of the above composition to identity map.

$$
\begin{aligned}
\left\|\zeta_{n} \circ \ldots \circ \zeta_{1}-\mathrm{id}\right\|_{\mathscr{C}^{1}}= & \| \zeta_{n} \circ \ldots \circ \zeta_{1}+\left(-\zeta_{n-1} \circ \ldots \circ \zeta_{1}+\zeta_{n-1} \circ \ldots \circ \zeta_{1}\right)+ \\
& +\left(-\zeta_{n-2} \circ \ldots \circ \zeta_{1}+\zeta_{n-2} \circ \ldots \circ \zeta_{1}\right)+\ldots+\left(-\zeta_{1}+\zeta_{1}\right)-\mathrm{id} \|_{\mathscr{C}^{1}} \\
\leq & \left\|\zeta_{n} \circ \ldots \circ \zeta_{1}-\zeta_{n-1} \circ \ldots \circ \zeta_{1}\right\|_{\mathscr{C}^{1}} \\
& +\left\|\zeta_{n-1} \circ \ldots \circ \zeta_{1}-\zeta_{n-2} \circ \ldots \circ \zeta_{1}\right\|_{\mathscr{C}^{1}} \\
& \vdots \\
& +\left\|\zeta_{2} \circ \zeta_{1}-\zeta_{1}\right\|_{\mathscr{C}}{ }^{1} \\
& +\left\|\zeta_{1}-\mathrm{id}\right\|_{\mathscr{C} 1} \\
\leq & \sum_{i=1}^{n} \varepsilon_{i}<\varepsilon .
\end{aligned}
$$

Step 6. Mapping in the limit Let

$$
\zeta: U \longrightarrow \mathbb{R}^{n} \text { be defined by } \zeta(u)=\lim _{n \rightarrow \infty} \zeta_{n} \circ \ldots \circ \zeta_{1}(u)
$$

Since $\mathscr{C}^{1}\left(U, \mathbb{R}^{n}\right)$ is a Banach space, if the sequence $\zeta_{n}$ is a Cauchy sequence then it will imply that $\zeta \in \mathscr{C}^{1}\left(U, \mathbb{R}^{n}\right)$. Let $\zeta_{n}^{\circ}:=\zeta_{n} \circ \ldots \circ \zeta_{1}$. Almost the same as in (38), for any $\varrho>0$

$$
\begin{aligned}
\left\|\zeta_{n}^{\circ}-\zeta_{m}^{\circ}\right\|_{\mathscr{C} 1} & =\left\|\zeta_{n}^{\circ}-\zeta_{n-1}^{\circ}+\zeta_{n-1}^{\circ}-\cdots-\zeta_{m+1}^{\circ}+\zeta_{m+1}^{\circ}-\zeta_{m}^{\circ}\right\|_{\mathscr{C}^{1}} \\
& \leq\left\|\zeta_{n}^{\circ}-\zeta_{n-1}^{\circ}\right\|_{\mathscr{C}^{1}}+\left\|\zeta_{n-1}^{\circ}-\zeta_{n-2}^{\circ}\right\|_{\mathscr{C}^{1}}+\cdots+\left\|\zeta_{m+1}^{\circ}-\zeta_{m}^{\circ}\right\|_{\mathscr{C}^{1}} \\
\text { from Eq.(34) } & \leq \varepsilon_{n}+\varepsilon_{n-1}+\cdots+\varepsilon_{m+1}+\varepsilon_{m}
\end{aligned}
$$

Since the series $\sum_{k} \varepsilon_{k}$ is convergent, one can find an integer $N$ such that for $n, m>N$ the condition

$$
\left\|\zeta_{n}^{\circ}-\zeta_{m}^{\circ}\right\|_{\mathscr{C} 1} \leq \varrho
$$

is satisfied, proving that $\zeta \in \mathscr{C}^{1}\left(U, \mathbb{R}^{n}\right)$. Notice that $D \zeta=$ id on the boundary because all $D \zeta_{n}$ are equal to the identity on the boundary.

Step 7. Construction for the whole set $U$ Having a construction on one element of the family $\mathfrak{U}$ we easily produce the sequence for the whole domain $U$, because on every element of the cover $\mathfrak{U}$ the modification can be done separately. From now on $U_{i}$ denotes an element of the 
family $\mathfrak{U}$ again and $\zeta_{i, n}$ is the mapping at the $n$ th-step on the element $U_{i}$. The mapping $\zeta_{i, n}$ can be extended with id to $U \backslash U_{i}$. Define the sequence $\left(\xi_{n}\right)_{n=1,2, \ldots}$ by

$$
\begin{aligned}
\xi_{1}= & \zeta_{1,1} \\
\xi_{2}= & \zeta_{1,2} \circ \zeta_{1,1} \circ \zeta_{2,1} \\
\xi_{3}= & \zeta_{1,3} \circ \zeta_{1,2} \circ \zeta_{1,1} \circ \zeta_{2,1} \circ \zeta_{1,1} \circ \zeta_{3,1} \\
\xi_{4}= & \zeta_{1,4}^{\circ} \circ \zeta_{2,3}^{\circ} \circ \zeta_{3,2}^{\circ} \circ \zeta_{4,1}^{\circ} \\
& \vdots \\
\xi_{n}= & \zeta_{1, n}^{\circ} \circ \zeta_{2, n-1}^{\circ} \circ \cdots \circ \zeta_{n-1,2}^{\circ} \circ \zeta_{n, 1}
\end{aligned}
$$

where $\zeta_{i, k}^{\circ}=\zeta_{i, k} \circ \zeta_{i, k-1} \circ \cdots \circ \zeta_{i, 1}$. The composition sign between functions in the last line is, in fact, a long list of identities because

$$
\operatorname{spt}\left(\zeta_{k, l}-\mathrm{id}\right) \cap \operatorname{spt}\left(\zeta_{m, n}-\mathrm{id}\right)=\varnothing \text { for } k \neq m .
$$

On every component $U_{i}$ sequence $\xi_{n}$ is converging to corresponding $\zeta$. After composition with the map $f$ the measure of $\Sigma \cap U_{i}$ is zero for every $i$.

\subsection{Reverse implication}

Reverse implication is an easy consequence of two facts. The first is that a rectifiable set can be filled up to a set of the measure zero with a countable family of $\mathscr{C}^{1}$ submanifolds and the second is the area formula.

Lemma 3.4 Let $\Sigma \subset U \subset \mathbb{R}^{n}$ be such that $\mathscr{H}^{m}(\Sigma)<\infty$ and assume that the interior of the set $\mathscr{A}(\Sigma)$ in the $\mathscr{C}_{=m}^{1}\left(U, \mathbb{R}^{n}\right)$ topology is empty, where as before

$$
\mathscr{A}(\Sigma):=\left\{f \in \mathscr{C}^{1}\left(U, \mathbb{R}^{n}\right) \mid \operatorname{dim} \operatorname{im} D f=m, \mathscr{H}^{m}(f(\Sigma))>0\right\} .
$$

Then $\Sigma$ is purely unrectifiable.

Proof We shall prove this lemma by contradiction. Assume that the interior of the set $\mathscr{A}(\Sigma)$ in $\mathscr{C}_{=m}^{1}\left(U, \mathbb{R}^{n}\right)$ is empty and $\mathscr{H}^{m}\left(\Sigma_{r}\right)>0$ where $\Sigma=\Sigma_{r} \cup \Sigma_{\text {unr }}$ and $\Sigma_{r}$ denotes rectifiable part of the set $\Sigma$. For a metric space $X, x \in X$ and a positive $\delta$ let

$$
B_{X}(x, \delta):=\left\{f \in X \mid\|f-x\|_{X}<\delta\right\} .
$$

Let $P_{m}: \mathbb{R}^{n} \rightarrow \mathbb{R}^{n}$ denotes the projection on the first $m$ coordinates. We shall find a mapping $\psi: \mathbb{R}^{n} \rightarrow \mathbb{R}^{n}$ and an open set $B_{\mathscr{C}=m}\left(P_{m} \circ \psi, \delta\right)$ such that the image of the set $\Sigma_{r}$ under any mapping from this ball has positive measure.

A rectifiable set can be covered up to a set of the $\mathscr{H}^{m}$ measure zero by countably many $m$ dimensional $\mathscr{C}^{1}$ submanifolds, see [8, Theorem 3]. Therefore there exists a family $\left\{M_{i}^{m}\right\}_{i \in \mathbb{N}}$ such that

$$
\mathscr{H}^{m}\left(\Sigma_{r} \backslash \bigcup_{i=1}^{\infty} M_{i}^{m}\right)=0,
$$

where $M_{i}^{m}$ is an $m$-dimensional $\mathscr{C}^{1}$ submanifold in $\mathbb{R}^{n}$. We may assume that

$$
\mathscr{H}^{m}\left(\Sigma_{r} \cap M_{1}^{m}\right)>0 .
$$


Let $x \in M_{1}^{m}$ be a point of the positive density of the set $\Sigma_{r} \cap M_{1}^{m}$. Since $M_{1}^{m}$ is a $\mathscr{C}^{1}$ submanifold then like in the paragraph before Corollary 2.1 there exists a $\mathscr{C}^{1}$-diffeomorphism $\psi$ and an open set $\tilde{B} \ni x$ such that

$$
\psi: \tilde{B} \rightarrow \mathbb{R}^{n} \text { and } \psi\left(\tilde{B} \cap M_{1}^{m}\right) \subset \mathbb{R}^{m} \times\left\{0^{n-m}\right\} .
$$

In other words, mapping $\psi$ straightens $M_{1}^{m}$ in some neighbourhood of the point $x$. By restricting to a ball $\bar{B}$ with $\mathscr{H}^{m}\left(\Sigma_{r} \cap M_{1}^{m} \cap B\right)>0$, compactly embedded in the set $\tilde{B}$, we obtain that both $\bar{B}$ and $\psi(\bar{B})$ are compact and $\psi, \psi^{-1}$ are $\mathscr{C}^{1}$ up to the boundary of the set $\bar{B}$ and $\psi(\bar{B})$ respectively. Let $M:=M_{1}^{m}$, then by Eq. (5) we have

$$
\begin{aligned}
\mathscr{H}^{m}\left(\psi\left(M \cap \Sigma_{r}\right)\right) & \geq\left(L_{\psi^{-1}}\right)^{-m} \mathscr{H}^{m}\left(\psi^{-1}\left(\psi\left(M \cap \Sigma_{r}\right)\right)\right) \\
& =\left(L_{\psi^{-1}}\right)^{-m} \mathscr{H}^{m}\left(M \cap \Sigma_{r}\right)>0 .
\end{aligned}
$$

where $L_{\psi^{-1}}$ is the Lipschitz constant for the mapping $\psi^{-1}$. Consider a set in the $\mathscr{C}_{=m}^{1}$ metric space consisting of mappings that are $\delta$ close to $P_{m} \circ \psi$ on the ball $\bar{B}$

$$
f \in B_{\mathscr{C}=m}\left(\bar{B}, \mathbb{R}^{n}\right)\left(P_{m} \circ \psi, \delta\right)
$$

For a compact $\bar{B}$ similarly to Eq. (34) one can see that precomposition with $\psi$ is continuous i.e.

$$
\begin{aligned}
\forall \varepsilon>0 \exists \delta>0 \quad\left\|f-P_{m} \circ \psi\right\|_{\mathscr{C}^{1}(\bar{B})} \leq \delta & \Longrightarrow\left\|f \circ \psi^{-1}-P_{m} \circ \psi \circ \psi^{-1}\right\|_{\mathscr{C}^{1}(\psi(\bar{B}))} \\
& =\left\|f \circ \psi^{-1}-P_{m}\right\|_{\mathscr{C}^{1}(\psi(\bar{B}))} \leq \varepsilon
\end{aligned}
$$

If we take $\varepsilon$ sufficiently small then the above imply that

$$
f \circ \psi^{-1}: \psi(\bar{B}) \cap\left(\mathbb{R}^{m} \times 0^{n-m}\right) \rightarrow \mathbb{R}^{n}
$$

is injective since it is $\varepsilon$ close to id $=\left.P_{m}\right|_{\mathbb{R}^{m} \times 0^{n-m}}$ in $\mathscr{C}^{1}$ metric. It is so because on every interval joining two points in the domain, function is equal to the integral of its derivative. If two points collapse to one under the above mapping then considering for example first coordinate we would have that integral of the derivative over the interval joining them is equal to zero. But this is impossible becasue $D\left(f \circ \psi^{-1}\right) . v$ is $\varepsilon\|v\|$-close to $v$, where $v$ is a vector joining those two points in the domain.

Now we use the area formula to prove that any such $g:=f \circ \psi^{-1}: \mathbb{R}^{m} \rightarrow \mathbb{R}^{n}$ has the property $\mathscr{H}^{m}\left(g\left(\psi\left(M \cap \Sigma_{r}\right)\right)\right)>0$. Let $\Sigma_{\psi}:=\psi\left(M \cap \Sigma_{r} \cap \bar{B}\right)$, then

$$
\begin{aligned}
\int_{g\left(\Sigma_{\psi}\right)} 1 d \mathscr{H}^{m} y & =\int_{g\left(\Sigma_{\psi}\right)} \mathscr{H}^{0}\left(\Sigma_{\psi} \cap g^{-1}(y)\right) d \mathscr{H}^{m}(y) \\
& =\int_{\Sigma_{\psi}} J_{g}(x) d x=\int_{\Sigma_{\psi}} \sqrt{\operatorname{det}\left(D g(x)^{\star} \cdot D g(x)\right)} d x
\end{aligned}
$$

where $A . B$ is usual multiplication of matrices $A \in M_{m \times n}$ and $B \in M_{n \times m}$. Now compare $D g$ to $D\left(P_{m}\right)=P_{m}$. Note that

$$
\begin{aligned}
D g(x)^{\star} \cdot D g(x)= & \left(P_{m}+\left(D g-P_{m}\right)\right)^{\star}(x) \cdot\left(P_{m}+\left(D g-P_{m}\right)\right)(x) \\
= & {\left[P_{m}\right]_{m}+\left(\left(\left[D g-P_{m}\right]^{m}\right)^{\star}+\left[D g-P_{m}\right]_{m}\right.} \\
& \left.+\left[D g-P_{m}\right]^{\star} \cdot\left[D g-P_{m}\right]\right)(x) \\
= & I_{m}+A_{1}(x)+A_{2}(x)+A_{3}(x) .
\end{aligned}
$$


where for $A \in M_{n \times m}$ the matrix $[A]_{m}$ is $m \times m$ matrix obtained by cutting off last $(n-m)$ rows and $[B]^{m}$ is again $m \times m$ matrix obtained from $B \in M_{m \times n}$ by cutting off last $(n-m)$ columns, and $I_{m} \in M_{m \times m}$ is identity matrix.

Note that if $\left\|g-P_{m}\right\|_{\mathscr{C} 1} \leq \varepsilon$ then $\left\|A_{i}\right\|$ for $i=1,2$ are smaller than $c \varepsilon$ and $\left\|A_{3}\right\|$ is smaller than $c \varepsilon^{2}$ with $c=c(n, m)$. The matrix $I_{m} \in M_{m \times m}$ is away from the set of matrices of rank less or equal than $m-1$. Hence if we pick $\varepsilon$ small enough

$$
I_{m}+\left(\left(\left[D g-P_{m}\right]^{m}\right)^{\star}+\left[D g-P_{m}\right]_{m}+\left[D g-P_{m}\right]^{\star} \cdot\left[D g-P_{m}\right]\right)(x) \in G l(m)
$$

Hence on the right hand side of Eq. (46) under the integral we have strictly positive function on a set of positive measure, therefore

$$
\left.\mathscr{H}^{m}\left(f\left(\Sigma_{r}\right)\right)\right)=\mathscr{H}^{m}\left(f \circ \phi^{-1} \circ \phi\left(\Sigma_{r}\right)\right)=\mathscr{H}^{m}\left(g\left(\Sigma_{\phi}\right)\right)>0 .
$$

It follows that for any $f$ form sufficiently small ball $B_{\mathscr{C}=m}^{1}(\bar{B})\left(P_{m} \circ \psi, \delta\right) \cap \mathscr{C}_{=m}^{1}\left(U, \mathbb{R}^{n}\right)$ the measure of the image of $\Sigma_{r}$ is positive hence we obtained a contradiction to our assumption about emptiness of the interior of the set $\mathscr{A}(\Sigma)$.

\subsection{Potential extension of the theorem}

Mappings with $m$ or lower rank.Under the conjecture

$$
\mathscr{C}_{\leq m}^{\infty}\left(U, \mathbb{R}^{n}\right) \stackrel{\text { dense }}{\subset} \mathscr{C}_{\leq m}^{1}\left(U, \mathbb{R}^{n}\right)
$$

our main theorem would be true for broader class of mappings $\mathscr{C}_{\leq m}^{1}\left(U, \mathbb{R}^{n}\right)$. The argument is as follows. Let $\left\|f_{\varepsilon}-f\right\|_{\mathscr{C}^{1}} \leq \varepsilon$ and $f_{\varepsilon} \in \mathscr{C}_{\leq m}^{\infty}$. Split the domain into two sets $U_{m}=$ $\left\{x \mid r\left(D f_{\varepsilon}(x)\right)=m\right\}$ and $U \backslash U_{m}$. On the set $U_{m}$ do the same procedure as for maps belonging to $\mathscr{C}_{=m}^{1}$, on the remaining set use the Sard theorem [5, Theorem 3.4.3]. It states that if $B=\left\{x \mid r\left(D f_{\varepsilon}(x)\right) \leq m-1\right\}$, the set $U \subset \mathbb{R}^{n}$ and the mapping $f$ is of class $k$, then

$$
\mathscr{H}^{m-1+(n-(m-1)) / k}(f(B))=0 .
$$

Apply it to $f_{\varepsilon}$ and notice that if $k>n-m+1$ then $m-1+(n-(m-1)) / k<m$ and then the $\mathscr{H}^{m}$ measure of the image of the whole set $B$ equals zero, hence $\mathscr{H}^{m}\left(f_{\varepsilon}(B \cap \Sigma)\right)=0$ as $f_{\varepsilon}$ belongs to any class $\mathscr{C}^{k}$. Therefore if Eq. (51) is true then the thing we have to do is to modify the map on the open set where the rank of the Jacobian matrix is equal to $m$.

Acknowledgements The author wants to thank Paweł Strzelecki and Sławomir Kolasiński for having drawn his attention to this problem. He would like to thank the audience of the "informal seminar" for constant support and useful discussions. He would also like thank two early stage readers: Kasia Mazowiecka and Maciek Zdanowicz for their invaluable help in improving the manuscript. The author was partially supported by NCN Grant no. 2013/10/M/ST1/00416 Geometric curvature energies for subsets of the Euclidean space

Open Access This article is distributed under the terms of the Creative Commons Attribution 4.0 International License (http://creativecommons.org/licenses/by/4.0/), which permits unrestricted use, distribution, and reproduction in any medium, provided you give appropriate credit to the original author(s) and the source, provide a link to the Creative Commons license, and indicate if changes were made.

\section{References}

1. Almgren, J.F.J.: Existence and regularity almost everywhere of solutions to elliptic variational problems among surfaces of varying topological type and singularity structure. Ann. Math. 2(87), 321-391 (1968)

2. Besicovitch, A.S.: On the fundamental geometrical properties of linearly measurable plane sets of points (III). Math. Ann. 116(1), 349-357 (1939). https://doi.org/10.1007/BF01597361 
3. David, G., Semmes, S.: Uniform rectifiability and quasiminimizing sets of arbitrary codimension. Mem. Am. Math. Soc. 144(687), viii + 132 (2000). https://doi.org/10.1090/memo/0687

4. Federer, H.: The $(\varphi, k)$ rectifiable subsets of $n$-space. Trans. Am. Soc. 62, 114-192 (1947)

5. Federer, H.: Geometric measure theory. Die Grundlehren der mathematischen Wissenschaften, Band 153. Springer, New York (1969)

6. Federer, H., Fleming, W.H.: Normal and integral currents. Ann. Math. 2(72), 458-520 (1960)

7. Feuvrier, V.: Un résultat d'existence pour les ensembles minimaux par optimisation sur des grilles polyédrales. Doctoral thesis(French), Mathématiques [math] Université Paris Sud. https://tel.archives-ouvertes. fr/tel-00348735/document (2008)

8. Hajłasz, P.: Sobolev mappings, co-area formula and related topics. In: Proceedings on analysis and geometry (Russian) (Novosibirsk Akademgorodok, 1999), pp. 227-254. Izdat. Ross. Akad. Nauk Sib. Otd. Inst. Mat, Novosibirsk (2000)

9. Mattila, P.: Geometry of sets and measures in Euclidean spaces: fractals and rectifiability. Cambridge studies in advanced mathematics. Cambridge University Press, Cambridge (1995). https://doi.org/10. 1017/CBO9780511623813

10. Pugh, H.: A localized Besicovitch-Federer projection theorem. ArXiv e-prints 1607, 01758 (2016)

11. Spivak, M.: Calculus on manifolds. A modern approach to classical theorems of advanced calculus. W. A. Benjamin Inc, New York (1965) 